\title{
Suppression of Retinal Neovascularization by Lentivirus-Mediated Netrin-1 Small Hairpin RNA
}

\author{
Huizhuo Xua Jiaolian Liu ${ }^{a}$ Siqi Xiong ${ }^{a}$ Yun-zheng Le ${ }^{b}$ Xiaobo Xia ${ }^{a}$ \\ ${ }^{a}$ Department of Ophthalmology, Xiangya Hospital, Central South University, Changsha, China; ${ }^{b}$ Departments of \\ Medicine, Endocrinology and Cell Biology and Harold Hamm Oklahoma Diabetes Center, University of Oklahoma \\ Health Sciences Center, Oklahoma City, Okla., USA
}

Key Words

Retinal neovascularization • Netrin-1 - Lentivirus · Small hairpin RNA

\begin{abstract}
Objectives: The function of netrin-1 in pathological angiogenesis and its role in retinal neovascularization were investigated in the retinas of oxygen-induced retinopathy (OIR) mice by inhibition of netrin-1. Methods: Expression of netrin-1 mRNA and protein in the retinas of OIR mice was analyzed by quantitative RT-PCR and immunoblotting. Inhibition of retinal neovascularization was achieved by lentivirus-mediated netrin-1 small hairpin RNA (shRNA) infection. Retinal neovascularization was examined by fluorescein angiography and quantification of preretinal neovascular nuclei in retinal sections. Results: Both mRNA and protein expression of netrin-1 were significantly upregulated in postnatal day 17 OIR mouse retinas. Treatment of OIR mice with specific lentivirus-mediated netrin-1 shRNA dramatically reduced neovascular outgrowth into the inner limiting membrane. Neovascular tufts and nonperfused areas were also reduced. Conclusions: High expression of netrin-1 was detected in the retina under ischemic conditions and played a
\end{abstract}

significant role in pathological retinal angiogenesis. Therefore, netrin-1 represents a potential therapeutic target for diabetic retinopathy, retinopathy of prematurity and other ocular neovascular diseases.

Copyright $\odot 2011$ S. Karger AG, Basel

\section{Introduction}

Pathological retinal neovascularization is one of the vision-threatening complications of ocular vascular diseases, including diabetic retinopathy, central retinal vein occlusion, neovascular glaucoma and retinopathy of prematurity. The pathological process comprises a series of events: degradation of extracellular matrix components, proliferation and migration of endothelial cells and tube formation. Many proangiogenic molecules have been demonstrated to play important roles in retinal neovascularization, including insulin-like growth factor, basic fibroblast growth factor, erythropoietin and vascular endothelial growth factor (VEGF) [1-4]. As a result of intensive biochemical and pharmacological studies, anti-VEGF agents have been widely used in clinical trials for blocking pathological retinal neovas-

\section{KARGER}

(ㄷ) 2011 S. Karger AG, Basel

Fax +41613061234

E-Mail karger@karger.ch

www.karger.com 
cularization [5]. However, VEGF blockade alone cannot completely eliminate pathological angiogenesis, suggesting that other angiogenic factors, such as hypoxiainducible factor (HIF) $1 \alpha$, erythropoietin and the recently described netrin-1 [6], may participate in these pathological processes.

Netrin-1, a $68-\mathrm{kDa}$ laminin-related molecule, was initially described as an axonal attractant growth factor that mediates guidance of developing axons via the DCC (deleted in colorectal cancer) receptor and UNC5 (uncoordinated five) families [7-9]. It has been shown that netrin-1 acts as guidance molecule for steering retinal ganglion cells into the optic nerve to form the optic fissure during embryonic retinal development $[10,11]$. In this process, retinal neurites respond to netrin- 1 in a dose-dependent manner, and the responses are reduced by blockade of DCC function. A lack of netrin-1 or DCC blocks the exit of retinal ganglion cell axons from the retina resulting in ectopic growth of retinal ganglion cells within the eye during embryonic retinal development $[12,13]$. The development of nerves and vessels occurs in parallel in two complex branching systems that exhibit many similarities in their networking and tissue distribution. Furthermore, their biogenesis is exquisitely regulated by common cues. To date, many molecules implicated in axonal guidance have been identified to regulate vessel sprouting, such as ephrins, slits, semaphorins and netrins. Netrin-1 has been reported to play important roles in embryonic and pathological angiogenesis [7].

However, these studies have produced contradictory results. Larrivée et al. [8] demonstrated that gene inactivation of UNC5B in mice was associated with increased angiogenesis suggesting antiangiogenic activity of netrin-1. Lu et al. [9] reported that netrin-1 induced filopodium retraction in wild-type mice but not in $\mathrm{UNC} \mathrm{B}^{-/-}$ mice, and knockdown of the netrin-1 ortholog in zebrafish caused abnormal branching in developing blood vessels, indicating that netrin-1 is a negative regulator of capillary branching via UNC5B. However, Park et al. [10] demonstrated that netrin-1 was capable of stimulating proliferation, migration and adhesion of vascular cells and also augmentation of the angiogenic activity of VEGF in vitro and in vivo. This study aimed to address these controversial issues by evaluation of netrin-1 mRNA and protein expression, and investigation of the effects of inhibition of retinal angiogenesis by efficient RNA interference (RNAi) of netrin-1 in the oxygen-induced retinopathy (OIR) mouse model.

\section{Materials and Methods}

\section{Cell Culture and Infection}

The mouse brain microvascular endothelial cell line (bEnd.3), purchased from the American Type Culture Collection (Manassas, Va., USA), was cultured in Dulbecco's modified Eagle's medium (Invitrogen, Carlsbad, Calif., USA) supplemented with $10 \%$ fetal bovine serum and $1 \%$ penicillin-streptomycin in a humidified incubator containing $5 \% \mathrm{CO}_{2}$ at $37^{\circ} \mathrm{C}$. Netrin-1 shRNA (G GGT GCC CTT CCA GTT CTA) was packaged by pGCSIL-GFP lentivirus (Gene Chem Co. Ltd., China). The scramble small hairpin RNA (shRNA) sequence was: G TGG CCC CGA AGC CTT TAC [14]. Infection with lentivirus was carried out according to the instructions provided by the manufacturer.

\section{Animal Model}

All animal experiments were carried out in accordance with the Association for Research in Vision and Opthalmology Statement for the Use of Animals in Ophthalmic and Vision Research. The murine OIR model has been previously described [15]. Briefly, C57BL/6J mice (purchased from Shanghai Laboratory Animal Center, Chinese Academy of Sciences) were exposed to $75 \pm 2 \%$ oxygen for 5 days from day 7 after birth (postnatal day, P) with the nursing mothers. On P12, the mice were removed from the hyperoxic environment and maintained under normal conditions until P17. Age-matched C57BL/6J mice maintained under normal oxygen conditions were used as controls (N17)

\section{Intravitreal Injections}

On P12, mice were anesthetized by intraperitoneal injection of $10 \%$ chloral hydrate $(0.03 \mathrm{ml})$. The lid fissure was opened using a scalpel blade, and $1 \mu$ l of netrin- 1 shRNA $\left(10^{8} \mathrm{TU}\right)$ or scrambled shRNA lentivirus was delivered into the vitreous cavity through a 32-gauge Hamilton needle and syringe. The eye was then repositioned and the lids were approximated over the cornea.

\section{Fluorescein Angiography}

Six P17 mice of each group were anesthetized and then perfused through the left ventricle with $1 \mathrm{ml}$ of PBS containing $50 \mathrm{mg}$ fluorescein-conjugated dextran (Sigma, St. Louis, Mo., USA). Eyes were enucleated and fixed in $4 \%$ paraformaldehyde for 20 $\mathrm{min}$. The retinas were dissected to remove the lens and cornea and were placed in $4 \%$ paraformaldehyde for a further $5 \mathrm{~min}$. The peripheral retina was cut in 4 places, and the retina was flat-mounted with glycerol/PBS (1:1).

\section{Western Blot Analysis}

The cultured cells and murine retinas were collected and lysed in lysis buffer (150 mM NaCl, $50 \mathrm{~mm}$ Tris-HCl, $\mathrm{pH} 7.4,2 \mathrm{~mm}$ EDTA, 1\% NP-40) containing protease inhibitors (Boehringer Mannheim, Germany). Total protein was resolved by SDS polyacrylamide gel electrophoresis and was then transferred onto a nitrocellulose membrane. The membrane was incubated with monoclonal anti-mouse netrin-1 (1:200 dilution; Sigma N3787 clone No. 158936) and monoclonal anti-mouse $\beta$-actin $(1: 10,000$ dilution; Sigma). Peroxidase-conjugated secondary antibodies were used as secondary detection reagents with an enhanced chemiluminescence kit (KeyGEN, China). Chemiluminescent signals were visualized by exposure to X-ray film. 
Fig. 1. Western blot analysis of netrin-1 expression in murine retinas: netrin-1 mRNA (a), representative Western blot (b), and netrin-1 protein (c). Results are expressed as means \pm SEM and analyzed by one-way ANOVA followed by the LSD $t$ test to evaluate significance ( $n=6 /$ group). Both mRNA and protein expression of netrin-1 were significantly elevated in the retinas of P17 OIR mice compared with age-matched N17 controls ( $\mathrm{p}<0.01)$.
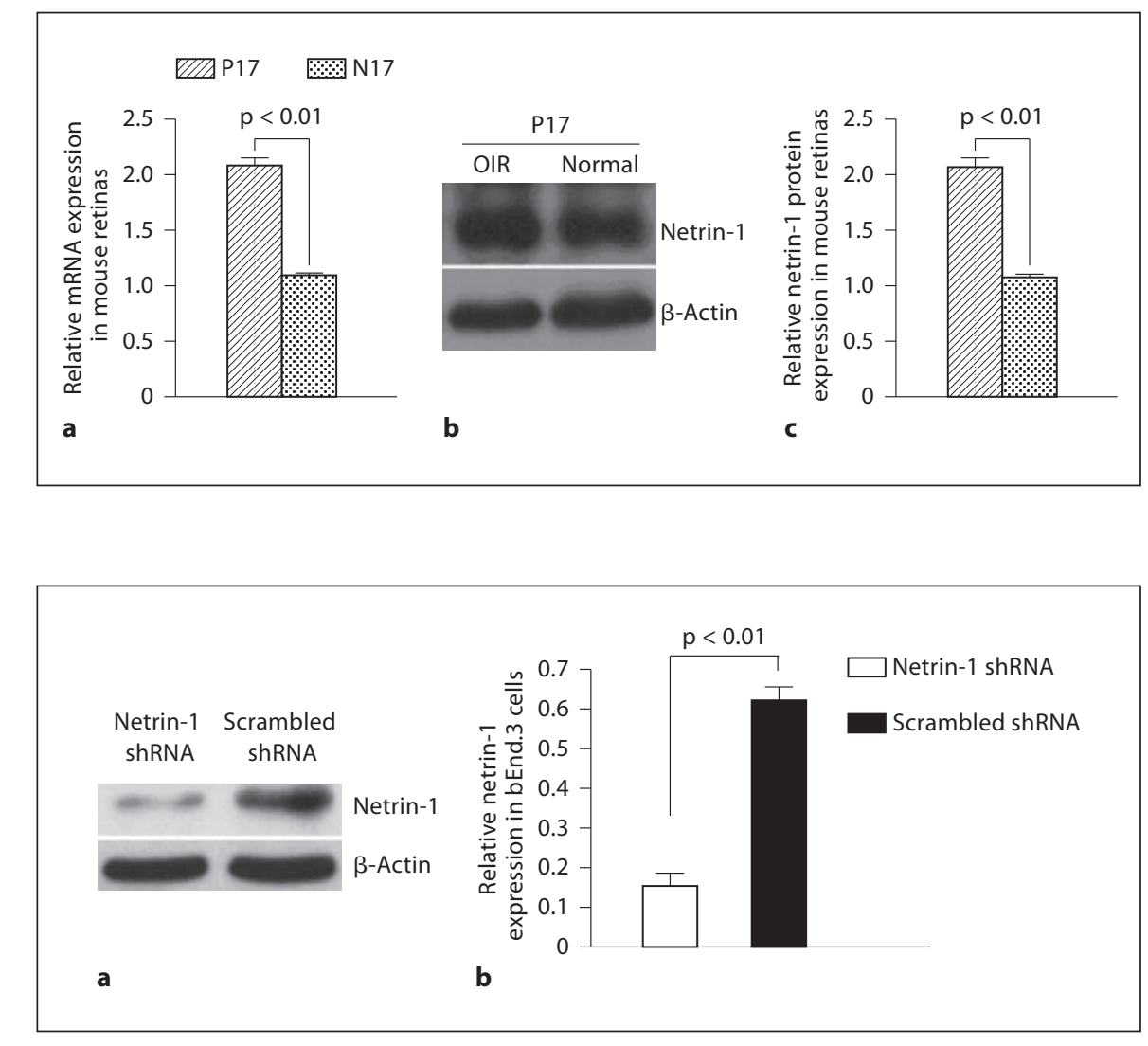

Fig. 2. Western blot analysis of the effect of RNAi on netrin-1 expression in bEnd.3 cells: representative Western blot (a) and relative expression of netrin-1 in bEnd. 3 cells (b). Results are expressed as means \pm SEM and analyzed by one-way ANOVA followed by the LSD $t$ test to evaluate significance ( $\mathrm{n}=3$ /group). Netrin-1 expression was significantly decreased in bEnd. 3 cells transfected with netrin-1 shRNA ( $\mathrm{p}<$ $0.01)$.

\section{Quantitative RT-PCR}

Total RNA was extracted and $1 \mu \mathrm{g}$ template was reverse-transcribed using the Revert Aid ${ }^{\mathrm{TM}}$ first-strand cDNA synthesis kit from MBI Biosystems (Fermentas, Copenhagen, Denmark). Realtime quantitative RT-PCR was performed on a 7900HT Fast RealTime PCR System apparatus (Applied Biosystems) using SYBR ${ }^{\circledR}$ Premix Ex Ta ${ }^{\mathrm{TM}}$ (TaKaRa, Shiga, Japan). The sequences of the netrin-1 primers were: CCA CCT CTG TGG ACC AGA AT (sense) and CTC CCT AGG GTG GGT AGG AG (antisense). The sequences of the $\beta$-actin primers were: CAG GAG ATG GCC ACT GCC GCA (sense) and CTC CTT CTG CAT CCT GTC AGC A (antisense).

\section{Cross-Sectional Analysis of Neovascular Tuft Formation}

All animals were sacrificed on P19. The eyes were enucleated and fixed in $4 \%$ paraformaldehyde for $24 \mathrm{~h}$, then embedded in paraffin. Serial sections $(5 \mu \mathrm{m})$ of whole eyes were cut sagittally through the cornea and parallel to the optic nerve. Sections were stained with hematoxylin and eosin. Ten nonserial sections were analyzed per eye. Sections including the optic nerve were excluded, and the nuclei of new vessels extending from the retina into the vitreous were counted in 360 sections.

\section{Statistical Analysis}

Results were expressed as means \pm SEM. One-way ANOVA followed by the LSD t test was used to evaluate significance. A p value $<0.05$ was considered statistically significant.

\section{Results}

\section{Upregulation of Netrin-1 in the Retina of OIR Mice}

Changes in netrin-1 mRNA and protein expression levels in the retina of OIR mice were examined. Netrin-1 mRNA and protein were significantly upregulated in P17 retinas of OIR mice compared with age-matched N17 controls (fig. 1).

\section{Suppression of Netrin-1 Expression in vivo and in vitro by Netrin-1 shRNA}

The effects of targeting netrin-1 with RNAi on netrin-1 expression were investigated by transfection of the netrin-1 shRNA or scrambled shRNA control into bEnd. 3 cells and the retinas of OIR mice. The level of netrin-1 protein expression was significantly downregulated in bEnd. 3 cells transfected with netrin-1 shRNA compared with that of cells transfected with scrambled shRNA (fig. 2). Similarly, intravitreal injection of netrin-1 shRNA on P12 resulted in clear downregulation of netrin-1 expression in the retinas of OIR mice on P17 (fig. 3). 
Fig. 3. Western blot analysis of the effect of RNAi on netrin-1 expression in murine retinas: representative Western blot (a) and relative expression of netrin-1 in murine retinas (b). Results are expressed as means \pm SEM and analyzed by one-way ANOVA followed by the LSD t test to evaluate significance ( $n=6 /$ group). Netrin-1 expression was significantly decreased in the retinas of P17 OIR mice transfected with netrin-1 shRNA compared with scrambled shRNA $(\mathrm{p}<0.01)$.

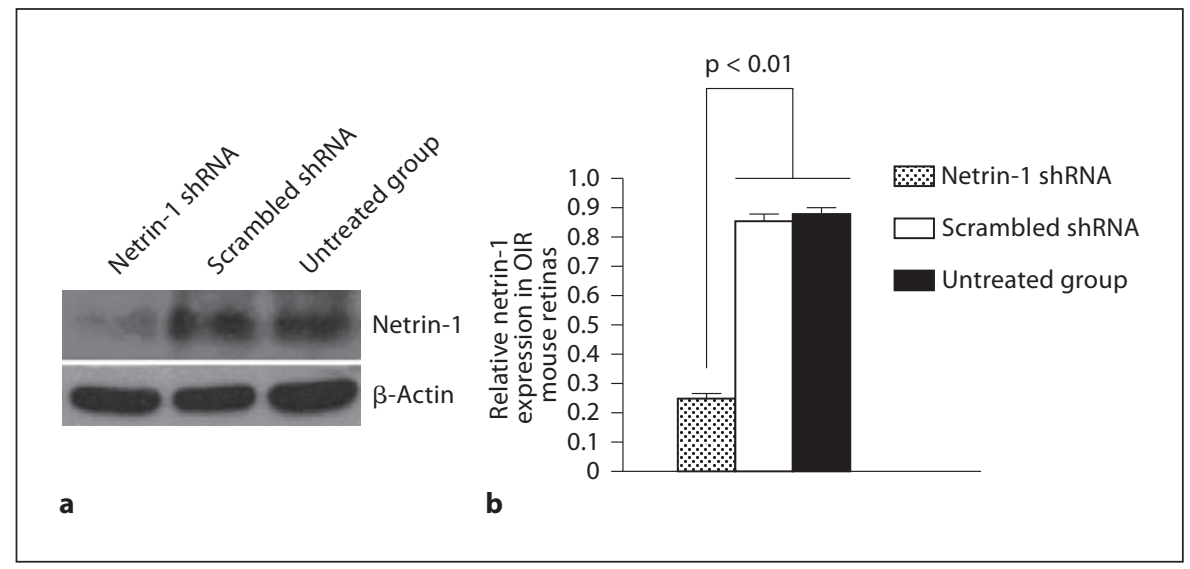

Suppression of Retinal Neovascularization by

Lentivirus-Mediated Netrin-1 shRNA

To determine the angiostatic effect of lentivirus-mediated netrin-1 shRNA on ischemia-induced retinal neovascularization, fluorescein angiography was performed in OIR mice on P17. Retinas of OIR mice transfected with scrambled shRNA appeared to have multiple neovascular tufts (hyperfluorescence) and central nonperfused areas. In contrast, fewer neovascular complexes and avascular regions were observed in the retinas of OIR mice transfected with lentivirus-mediated netrin-1 shRNA (fig. 4). To further confirm the inhibitory effects of netrin-1 shRNA on angiogenesis, retinal neovascularization was assessed histologically by counting the neovascular nuclei protruding into the vitreous cavity. The average numbers of neovascular nuclei were $9.8 \pm 1.7$ in the retinas of OIR mice transfected with lentivirus-mediated netrin-1 shRNA and $20.3 \pm 3.4$ in the retinas of OIR mice injected with scrambled shRNA, thus representing approximately a $50 \%$ reduction in retinal neovascularization (fig. 5).

\section{Discussion}

In the OIR mouse model, the retinal vasculature initially underwent reversible central vasoconstriction followed by nonperfusion on P7 when exposed to hyperoxia, then more peripheral vessels were spared, a small avascular zone appeared at the ora serrata, larger central radial vessels became tortuous and engorged on P14, and retinal neovascularization occurred extensively during P17 and P21 [15]. In this study, expression of both netrin-1 mRNA and protein was highly upregulated in the retinas of P17 OIR mice compared with controls, which is consistent with the results Tian et al. [16] presented, indicating that the mRNA and protein levels of netrin-1 were upregulated simultaneously with the increase in retinal neovascularization. This suggests that netrin-1 may play an important role in pathological retinal neovascularization, and its upregulation may promote angiogenesis. Previous studies have demonstrated a much stronger expression of netrin-1 in OIR retinas, mainly localized to the ganglion cell layer and the inner nucleic layer. Furthermore, it was observed that areas of neovascular vessels had broken through the inner limiting membrane in the retina [17]. Therefore, it was hypothesized that netrin-1, a laminin protein expressed at high levels in the ganglion cell layer and inner nucleic layer in the retina, then secreted to the epiretina and further to the vitreous cavity, is involved in retinal neovascularization and is important in pathological angiogenesis in the retina. HIF is a transcription factor found in mammalian cells cultured under reduced oxygen tension and plays a key role in the cellular response to hypoxia. Many genes involved in different steps of retinal angiogenesis are found to be regulated by HIF-1. Examples include VEGF, VEGF receptor 1, platelet-derived growth factor $\beta$, endothelin-1, erythropoietin and inducible NO synthase [6]. Expression of netrin-1 was significantly increased in the murine retinas of OIR. Further experiments are necessary to determine the relationship between netrin- 1 and HIF-1.

RNAi is a sequence-specific RNA degradation process that is conserved in eukaryotes and mediates target-specific RNA sequence degradation through a dsRNA-induced silencing complex [18]. Many delivery systems such as retroviral, adenoviral and lentiviral vectors have 
Fig. 4. Fluorescein angiography demonstrating the effect of the lentivirus-mediated netrin-1 shRNA on retinal neovascularization in OIR mice: retinas injected with scrambled shRNA (a) and retinas transfected with netrin-1 shRNA (b). The numbers of neovascular tufts (marked by white arrows in a) were markedly reduced, and nonperfusion areas were significantly diminished in the retinas transfected with netrin-1 shRNA.
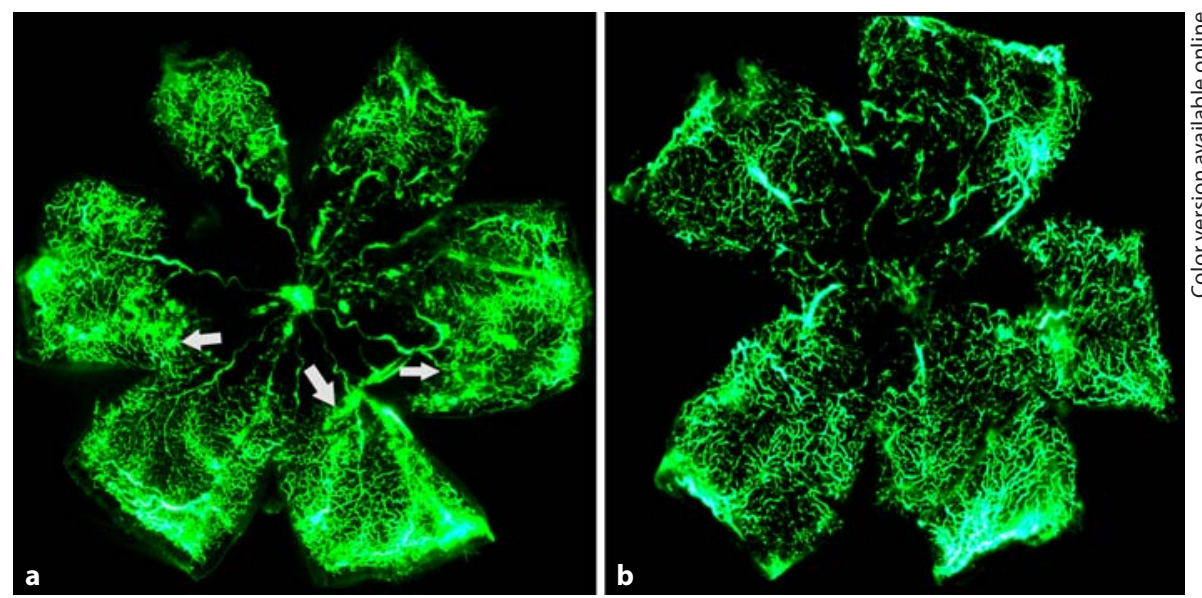
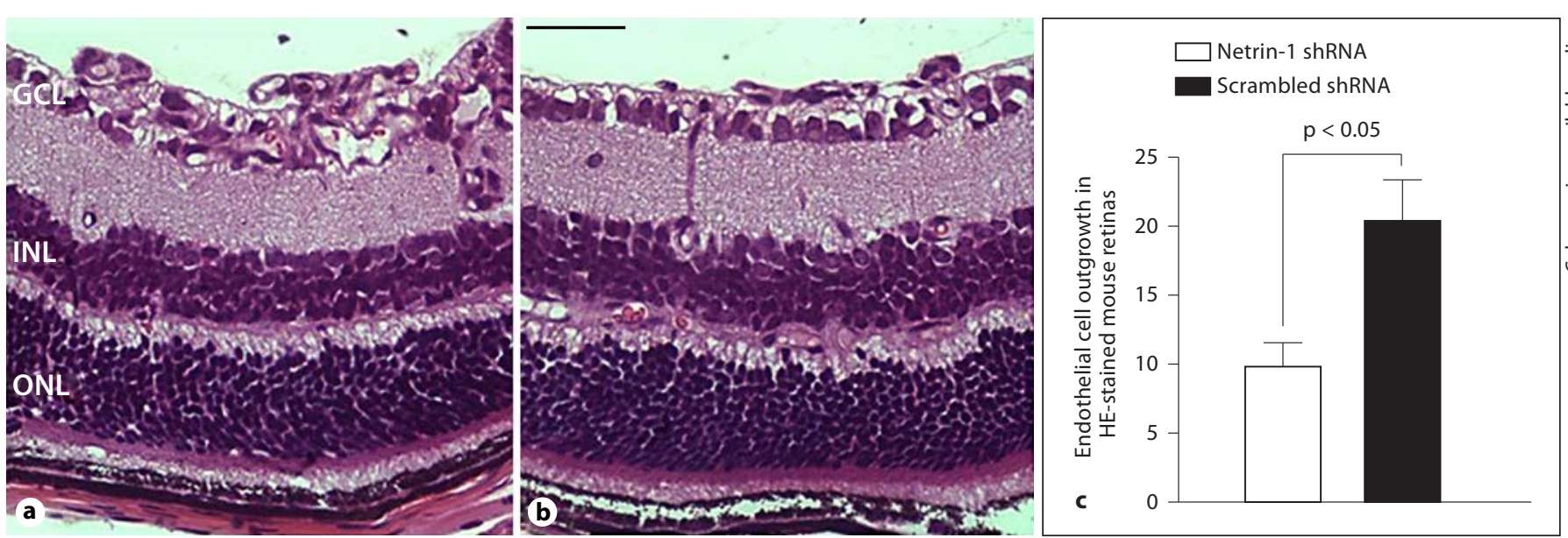

Fig. 5. Inhibition of ischemia-induced retinal neovascularization by netrin-1 shRNA. a A P17 OIR retina injected with scrambled shRNA on P12. b A P17 OIR retina transfected with netrin-1 shRNA. c Quantification of retinal neovascularization
( $\mathrm{n}=6$ /group). Netrin-1 shRNA resulted in approximately $50 \%$ reduction of preretinal neovascularization. GCL $=$ Ganglion cell layer; $\mathrm{INL}=$ inner nuclear layer; $\mathrm{ONL}=$ outer nuclear layer. Scale bar $=50 \mu \mathrm{m}$. recently been explored for specific and efficient inhibition of a target gene [19]. The lentiviral vectors used in this study were RNA polymerase-III-based shRNA expression vector systems that have been established to induce RNAi in mammalian cells. This system mediates reproducible stable and long-term transfection and gene silencing via integration of the specific RNAi sequence into the target gene sequence [20]. As shown in this study, netrin-1 expression decreased dramatically in vitro and in vivo following netrin-1 shRNA infection. These results suggest that lentivirus-mediated shRNA netrin-1 knockdown is efficient and reliable.

Retinal neovascularization and nonperfused areas were clearly reduced by netrin-1 shRNA transfection in the OIR model. Furthermore, new vessels and endothelial cell outgrowth of the inner limiting membrane in the retinas treated with netrin-1 shRNA were also significantly reduced. These results indicate that inactivation of netrin-1 expression by RNAi reduces pathological retinal neovascularization in OIR mice, and they are in accordance with a previous study demonstrating that silencing netrin- $1 \alpha$ expression results in the disappearance of parachordal vessels, longitudinal vessels adjacent to the notochord along the zebrafish trunk [7]. Other research has also shown that low concentrations of netrin-1 promote migration, proliferation and tube formation in human umbilical vein endothelial cells [21] and reduce endothelial cell apoptosis [22]. Netrin-1 is a po- 
tent mitogen and chemoattractant for both endothelial cells and vascular smooth muscle cells with a specific activity comparable to that of VEGF. Moreover, netrin-1 is capable of inducing sprout angiogenesis independently and of augmenting the VEGF-induced sprout angiogenesis in the murine corneal limbus. All of these observations suggest that netrin-1 functions as a proangiogenic factor in pathological retinopathy. Futhermore, Yang et al. [21] verified that netrin-1 promotes placental vascular growth in a matrigel plug assay and an in vitro rat aortic ring assay. It was observed that netrin-1 strongly stimulated in vivo neovascularization in a mouse matrigel plug and induced sprouting of endothelial cells in vivo in rat aortic rings.

It is hypothesized that the observed netrin-1 knockdown in retinas in OIR mice reduced pathological neovascularization mediated by blockade of proangiogenic pathways. It can be speculated that netrin-1 exerts its function in pathological angiogenesis through two possible mechanisms. Firstly, it may promote angiogenesis by induction of NO production via activation of the DCCdependent ERK1/2-endothelial NO synthase feed-forward pathway resulting in endothelial cell proliferation in response to NO-stimulated survival through a VEGFdependent mechanism [23]. Alternatively, netrin-1 may directly stimulate antiapoptotic signaling cascades in en- dothelial cells by inhibition of death-associated protein kinase, which inhibits the apoptosis of endothelial cells [24].

The exact functions of netrin-1 differ according to tissue type [25], developmental stage [26], pathological circumstances, and especially its receptor-signaling pathways [27]. However, these data demonstrate that ischemia-induced retinal neovascularization was successfully treated with netrin-1 RNAi in the OIR mouse model. This suggests that netrin-1 functions as an important proangiogenic growth factor in the pathological neovascularization of OIR. Therefore, netrin-1 represents a novel target for the therapy of diabetic retinopathy, retinopathy of prematurity and other ocular neovascular diseases.

\section{Acknowledgement}

This study was supported by the national Natural Science Foundation of China, grant 30872822, and NIH grant R01EY20900.

\section{Disclosure Statement}

All authors have no proprietary interest.

\section{References}

1 Watanabe D, Suzuma K, Matsui S, Kurimoto M, Kiryu J, Kita M, Suzuma I, Ohashi H, Ojima T, Murakami T, Kobayashi T, Masuda S, Nagao M, Yoshimura N, Takagi H: Erythropoietin as a Retinal Angiogenic Factor in Proliferative Diabetic Retinopathy. N Engl J Med 2005;353:782-790.

-2 Pe'er J, Shweiki D, Itin A, Hemo I, Gnessin H, Keshet E: Hypoxia-induced expression of vascular endothelial growth factor by retinal cells is a common factor in neovascularizing ocular diseases. Lab Invest 1995;72:638-645.

- 3 Aiello LP, Northrup JM, Keyt BA, Takagi H, Iwamoto MA: Hypoxic regulation of vascular endothelial growth factor in retinal cells. Arch Ophthalmol 1995;113:1538-1544.

-4 Sondell M, Sundler F, Kanje M: Vascular endothelial growth factor is a neurotrophic factor which stimulates axonal outgrowth through the flk-1 receptor. Eur J Neurosci 2000;12:4243-54.

-5 Rosenfeld PJ, Rich RM, Lalwani GA: Ranibizumab: phase III clinical trial results. Ophthalmol Clin North Am 2006;19:361-372.
-6 Xia XB, Xiong SQ, Xu HZ, Jiang J, Li Y: Suppression of retinal neovascularization by shRNA targeting HIF-1 $\alpha$. Curr Eye Res 2008;33:892-902.

7 Wilson BD, Li M, et al: Netrins promote developmental and therapeutic angiogenesis. Science 2006;313:640-644.

8 Larrivée B, Freitas C, Trombe M, Lu X, Delafarge B, Yuan L, Bouvrée K, Bréant C, Del Toro R, Bréchot N, Germain S, Bono F, Dol F, Claes F, Fischer C, Autiero M, Thomas JL, Carmeliet P, Tessier-Lavigne M, Eichmann A: Activation of the UNC5B receptor by netrin-1 inhibits sprouting angiogenesis. Genes Dev 2007:21:2433-2447.

$\checkmark 9$ Lu X, Le Noble F, Yuan L, Jiang Q, De Lafarge B, Sugiyama D, Bréant C, Claes F, De Smet F, Thomas JL, Autiero M, Carmeliet P, TessierLavigne M, Eichmann A: The netrin receptor UNC5B mediates guidance events controlling morphogenesis of the vascular system. Nature 2004;432:179-186.
10 Park KW, Crouse D, Lee M, Karnik SK, Sorensen LK, Murphy KJ, Kuo CJ, Li DY: The axonal attractant netrin-1 is an angiogenic factor. Proc Natl Acad Sci USA 2004;101: 16210-16215.

11 Cristofaro B, Emanueli C: Possible novel targets for therapeutic angiogenesis. Curr Opin Pharmacol 2009;9:102-108.

12 Deiner MS, Kennedy TE, Fazeli A, Serafini T, Tessier-Lavigne M, Sretavan DW: Netrin-1 and DCC mediate axon guidance locally at the optic disc: loss of function leads to optic nerve hypoplasia. Neuron 1997;19:575-589.

13 Oster SF, Deiner M, Birgbauer E, Sretavan DW: Ganglion cell axon pathfinding in the retina and optic nerve. Semin Cell Dev Biol 2004;15:125-136.

14 Furne C, Rama N, Corset V, Chédotal A, Mehlen P: Netrin-1 is a survival factor during commissural neuron navigation. Dev Biol 2008,105:14465-14470.

15 Smith LE, Wesolowski E, McLellan A, Kostyk SK, D'Amato R, Sullivan R, D'Amore PA: Oxygen-induced retinopathy in the mouse. Invest Ophthalmol Vis Sci 1994;35: 101-111. 
-16 Tian XF, Xia XB, Xiong SQ, Jiang J, Liu D, Liu JL: Netrin-1 overexpression in oxygen-induced retinopathy correlates with breakdown of the blood-retina barrier and retinal neovascularization. Ophthalmologica 2011; 226:37-44.

17 Ellezam B, Selles-Navarro I, Manitt C, Kennedy TE, McKerracher L: Expression of netrin-1 and its receptors DCC and UNC-5H2 after axotomy and during regeneration of adult rat retinal ganglion cells. Exp Neurol 2001;168:105-115.

- 18 Abbas-Terki T, Blanco-Bose W, Déglon N, Pralong W, Aebischer P: Lentiviral-mediated RNA interference. Hum Gene Ther 2002; 13:2197-2201.

-19 Manjunath N, Wu H, Subramanya S, Shankar P: Lentiviral delivery of short hairpin RNAs. Adv Drug Deliv Rev 2009;61:732745 .
20 Nishitsuji H, Ikeda T, Miyoshi H, Ohashi T, Kannagi M, Masuda T: Expression of small hairpin RNA by lentivirus-based vector confers efficient and stable gene-suppression of HIV-1 on human cells including primary non-dividing cells. Microbes Infect 2004;6 76-85.

21 Yang Y, Zou L, Wang Y, Xu KS, Zhang JX Zhang JH: Axon guidance cue netrin-1 has dual function in angiogenesis. Cancer Biol Ther 2007;6:743-748.

22 Castets M, Coissieux MM, Delloye-Bourgeois C, Bernard L, Delcros JG, Bernet A, Laudet V, Mehlen P: Inhibition of endothelial cell apoptosis by netrin-1 during angiogenesis. Dev Cell 2009;16:614-620.

23 Nguyen A, Cai H: Netrin-1 induces angiogenesis via a DCC-dependent ERK1/2-eNOS feed-forward mechanism. Proc Natl Acad Sci USA 2006;103:6530-6535.
24 Castets M, Mehlen P: Netrin-1 role in angiogenesis: to be or not to be a pro-angiogenic factor? Cell Cycle 2010;9:1466-1471.

25 Freitas C, Larrivée B, Eichmann A: Netrins and UNC5 receptors in angiogenesis. Angiogenesis 2008;11:23-29.

-26 Bouvrée K, Larrivée B, Lu X, Yuan L, De Lafarge B, Freitas C, Mathivet T, Bréant C, Tessier-Lavigne M, Bikfalvi A, Eichmann A, Pardanaud L: Netrin-1 inhibits sprouting angiogenesis in developing avian embryos. Dev Biol 2008;318:172-183.

27 Larrivée B, Freitas C, Trombe M, Lu X, De Lafarge B, Yuan L, Bouvrée K, Bréant C, Del Toro R, Bréchot N, Germain S, Bono F, Dol F, Claes F, Fischer C, Autiero M, Thomas JL, Carmeliet P, Tessier-Lavigne M, Eichmann A: Activation of the UNC5B receptor by netrin-1 inhibits sprouting angiogenesis. Genes Dev 2007;21:2433-2447. 